\section{Nicola L. Rizzi Valerio Varano}

\title{
Leo: a Multimedia Tale of Structural Mechanics
}

The authors have devised a method for teaching structural mechanics articulated in three phases: observations (the description of mechanical phenomena, increasingly complex, selected with regards to their pertinence of the problem that one wants to affront, and their efficiency); modeling (the construction of a physical-mathematical model that takes into account its formal content and stresses its importance as an instrument and has the potential for other applications); design (suggestion of cues for applications stimulate the student to exercise his creative imitation). What is proposed to the student is not so much a set of notions, as a method and set of instruments for selecting experiences (for example previous design solutions) to the end of evaluating their repeatability in diverse situations, by means of a physical-mechanical reading which comes from phenomena which one finds in daily life. "Leo" was created as a teaching instrument which is presented as a tale in the form of a hypertext

\section{Introduction}

The courses which directly confront design themes of the Italian Schools of Architecture, in particular architectural design, utilize a global integrated method ${ }^{1}$ that exposes the student to the complexity of a project by stimulating him to find individually his own learning approach and master it. The instrument of communication, that is the teaching method utilized, is essentially figurative (graphic representations, 3D models).

On the other hand, structural mechanics is taught prevalently by means of typical instruments of a formalized discipline, that is, a demonstrative or deductive method, and the language of mathematics.

The co-existence of these two approaches poses a delicate teaching problem, which if tackled without caution and the necessary attention, can cause the students to perceive the presence of two routes that evolve in parallel, so that the students, disoriented by the differences, fail to grasp the meaning, and perhaps convince themselves that they are being subjected, for inexplicable academic reasons, to a useless and boring assignment.

If on the one hand this can obscure, in the student's mind, the potential application of the knowledge of structural mechanics (which becomes merely material for specialists, on the other hand, it can also engender the deep-rooted ideology of thought (dense with a consolatory confusion) that in the end scientific disciplines do not have much to do with architecture.

Instead, we are convinced that the co-existence of such different approaches will be a happy prerogative of the Architectural Faculty and constitute a precious resource to resist the insistent requests for a specialized education that often is unacceptable to the University.

But how can one preserve the specificity of scientific culture and methods and achieve the objective of a strong integration of structural disciplines in the formation of architectural students?

Leo, the multimedia tale which we are presenting in this document, would like to contribute to this debate. 


\section{Methodological problems}

When confronting the study of formalized disciplines, architectural students prevalently face the following problems:

1. Difficulty in managing abstract concepts (axioms of scientific theory);

2. Little interest in formal rigor (mathematical language and its esthetics);

3. Difficulty in following a pure demonstrative approach (mathematical methods).

Students have difficulty in confronting the heart of abstract scientific theories and have a strong need for concrete examples.

This request is not unusual, since every scientific theory-and in particular those with strong technological implications - have some rules of correspondence ${ }^{2}$ which tie the constructs to the behavior of real objects. The objects of mechanics, and in particular those of structural mechanics, are especially rich in cues and suggestions, with a range of complexity from the simple facts of daily experiences to complex technical applications. We believe that by drawing from the appropriate repertoire we can significantly reduce any of learning difficulties mentioned above. In particular, we propose a teaching approach which meets the following requirements:

1. The abstract route which leads to the construction of theoretical models should be presented with a constant reference to selected examples.

2. It is beneficial when these examples are presented with illustrations which constitute an aid to the understanding of the formal language without taking its place.

Once the results of the theory are presented, the nature of the 'Rule of Interpretation' of the behavior of real objects must be stressed so that the student can utilize their worth in predicting outcomes of in the design process.

These considerations suggest a teaching method which is articulated in the following three phases:

- observations;

- modeling;

- design.

By 'observations', we mean the description of mechanical phenomena, increasingly complex, selected with regards to their pertinence of the problem that one wants to affront, and their efficiency. One needs to induce the student to feel the need to form a reference point (or a theory) and push him to follow the process of abstractions or generalization which must be progressive and in some way personal, then to guide him in the construction of a physical-mathematical model that takes into account its formal content and stresses its importance as an instrument and has the potential for other applications. The suggestion of cues for applications stimulates the student to exercise his creative imitation, which constitutes the design phase. He is prompted to not only verify the feasibility of his design ideas, but to explain it overall using a pre-figurative (pre-vision) mental design.

What we intend to propose to the student is not so much a set of notions, as a method and set of instruments for selecting experiences (for example previous design solutions) to the end of evaluating their repeatability in diverse situations, by means of a physical-mechanical reading which comes from phenomena which one finds in daily life.

100 NicOla L. RIZZI AND VALERIO VARANO - Leo: A Multimedia Tale of Structural Mechanics 
For this reason, Leo was created as a teaching instrument which is presented as a tale in the form of a hypertext.

\section{Leo is a multimedia tool}

The tale opens with an image of a student (Leo) who has begun studying for an architectural degree (armed with the only knowledge of mechanics learned during high school). While he is doing a simple assignment of an architectural design, he decides to construct a model and in doing so, he constructs the structure of a building without thinking too much about it (see Fig. 1).

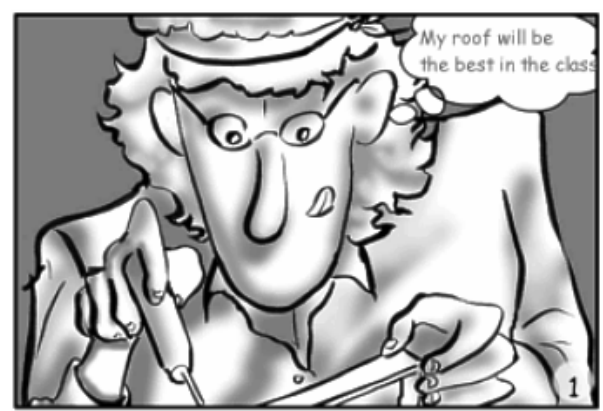

Fig. 1

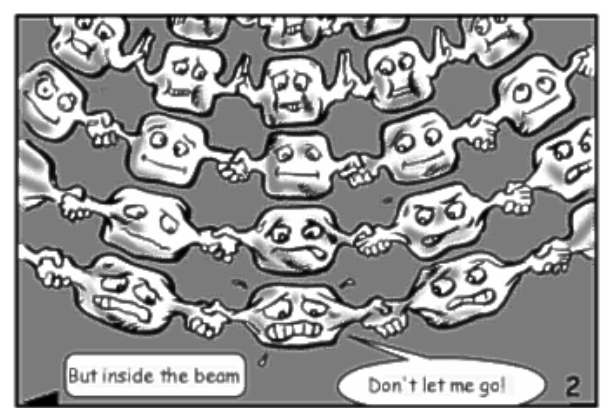

Fig. 2

Incited by his own curiosity to test the resistance, he is a little puzzled to find that his construction is not able to resist modest even stress tests; in other words, the model has difficulty staying erect. He immediately makes some spontaneous attempts to improve the situation, but these attempts are substantially inefficient.

This gives him the sensation of having ignored a relevant aspect of the project and of not possessing the adequate conceptual instruments to affront the problem or to even consider it. Zooming into the material itself (see fig. 2), shows how miniscule parts are lined up to represent the status of internal stresses, emphasizing the change of requirements and the necessity to learn a behavioral mechanical model of structures and the materials of which it is composed.

An intellectual journey begins during which, through successive abstractions, will lead to his learning physical-mathematical models which will progressively allow him to affront the design themes in a more knowing way. The intellectual journey is expressed in the metaphor of a journey in 'Structural Wonderland', during which he will meet with a series of constructions that are utilized to illustrate the concepts of structural mechanics. The narrative is presented as a comic strip. The rest is presented using a series of films which start from an example and pass to a model in $3 \mathrm{D}$ graphics, and conclude with the true mathematical model.

Computer simulations have revealed themselves to be powerful and versatile instruments to represent the mechanism of progressive abstraction, the multiple possibilities of rendering, from photo realism to pure geometry, in fact, permits us to highlight aspects one at a time which are considered pertinent (that is, ones that are chosen as) evidence in the phenomenon being studied.

\section{The contents}

The journey corresponds to turning pages in a textbook in which the chapters correspond to each stage of the journey (Fig. 5). The choice of themes and their articulation corresponds to the program of one or more modules or one course. 
We have worked on a program which we think constitutes the minimum level of knowledge which must be mastered by the end of the three-year Italian program in Architecture. The corresponding hypothetical book contains the following chapters (in parenthesis appears the name of the stage in the tale):

- Introduction (Entrance into Wonderland);

- Kinematics of Rigid Bodies (Boy Scout camp);

- Statics of Rigid Bodies: (The Sacred Area of Megaliths);

- Beam Theory (Construction site).

Naturally this can be integrated or modified in order to adapt to different teaching approaches.

In the initial stage, some concrete fundamentals are introduced in an intuitive manner of the structural mechanics.

Concepts of body, motion, deformation, force as an interaction between bodies, equilibrium, material behavior (strength, stiffness) and others, are introduced by means of a series of metaphors (such as team playing, synonymous of structural organizations), almost all of which are acted out on anthropomorphized structural elements, with the aim of relating physical concepts to the life experiences of the student himself (see figs. 2 - 4).

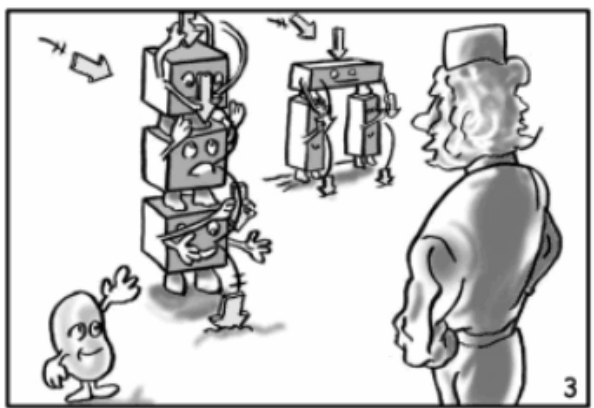

Fig. 3

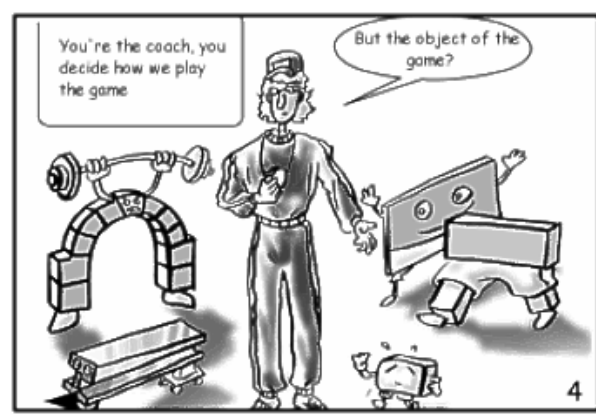

Fig. 4

At first glance, it might seem contradictory to begin an exposition which declares itself to be substantially empirically inductive by treating general concepts. In reality, they are presented not as elements of a defined theory, but rather to help the student take cues from mechanical experiences that are certainly part of his life experience in order to begin a process of selecting pertinent phenomenon.

\section{Analysis of a chapter: kinematics of a rigid body}

But the heart of the hypertext is made up of the examples. From a general view of the world of structures it is possible to access several sections, each one of which contains a structural example utilized to introduce some concept.

All of the examples are analyzed according to the same pattern, which utilizes substantially three areas: the screen on which the films are visualized; the dialogue box which accompanies the films; and the small bar with buttons that activate the films (see fig. 5). Each film relates to one of the three phases of the presentation:

102 NicOLA L. RIZZI AND VALERIO VARANO - Leo: A Multimedia Tale of Structural Mechanics 


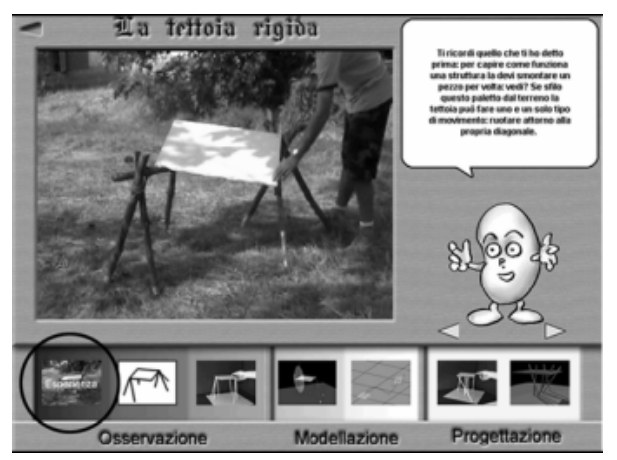

Fig. 5

Observation is subdivided into three parts: Experience, Rough Simulation, Experiment.

Modeling is subdivided into two parts: Geometric Modeling and Algebraic Modeling.

Design is subdivided into two parts: Simple Design and Complex Design.

To illustrate the significance of the single phases we shall refer to the first example, utilized for the presentation of the 'Kinematics of a rigid body' (linearized).

(Note that in the following the text in italics refers to the specific example while the photographs are sample frames of the films in the hypertext.)

5.1 Observation: From experience to experimentation. The Experience phase corresponds to a live film which demonstrates some behavior of a simple real object.

In the case study, the filming refers to a small structure made manually with sticks and rope as an example of an elementary construction such as a roof (see fig. 6).

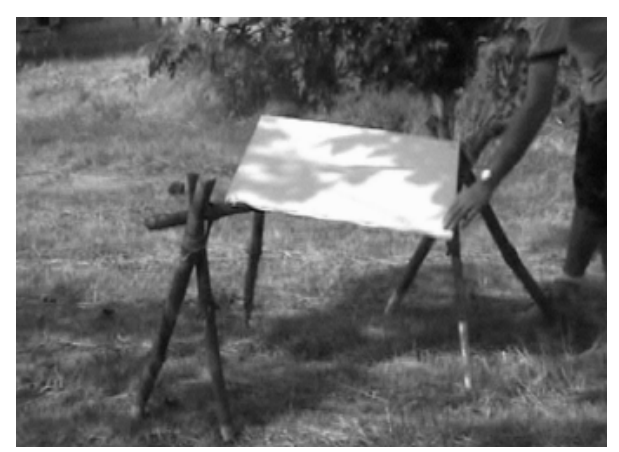

Fig. 6

Removing and adding sticks (constraints) shows the kinematic behavior of construction, prompting the reader to ask questions.

However, in order to understand the behavior of an existing structure in general one can not cut it into pieces! Therefore, a Rough Simulation is proposed (see fig. 7) as an intermediate phase which permits, by means of an initial abstraction, the identification of the pertinent characteristics and a study of them by means of a simulation in a laboratory.

NEXUS NETWORK JOURNAL - VOL. 7 NO. 1, 2005103 


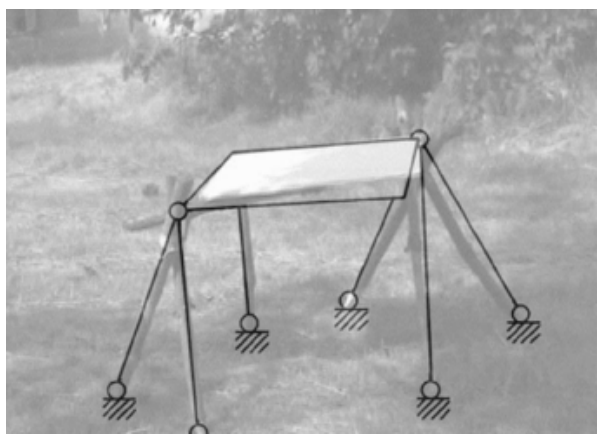

Fig. 7

Films in the Experiment stage describe the phenomena in a clear and qualitative manner.

Constraints are removed or rearranged one at a time, hypothesizing a classification of residual movements, questioning the relationship that exists between the number of constraints and classes of residual movements, (the protagonist does not still possess the notion of degree of freedom). Obviously, the result is that the number of constraints necessary to block the system is 6 and that this number is also sufficient, except when the constraints are put together badly (singular cases: linearly dependent constraints). Naturally, without a theory it is impossible to foresee the occurrence of such situations other than by making experimental attempts.

The proposed experiments were selected so as to be easily reproduced by students even at home, with inexpensive materials and without complex instruments (see fig. 8).

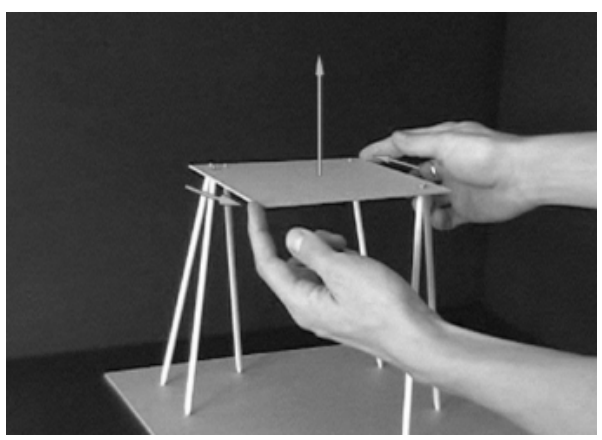

Fig. 8

\subsection{The mathematical model}

5.2.1 Geometry. At this point, the protagonist, stimulated by previous qualitative observations, is guided through the modeling phase.

Here he discovers that geometry, with which a student of architecture has a certain familiarity, is an useful instrument for constructing a model with a sufficient predictability that gives a powerful support for the imagination and design process. The films of this section are realized with computer graphics and, by means of a schematic reproduction of the analyzed structure, give a geometric explanation of the behavior evidenced in Experiment phase.

104 NiCOLA L. RizZI AND VALERIO VARANO - Leo: A Multimedia Tale of Structural Mechanics 
In reality, an inverse problem to that which is affronted in experiments is proposed: one starts from a free rigid body (e.g., a square) and one asks how many constraints do you need to block every possible movement?' From the initial idea of fixing the coordinates of three points on a body, one arrives at the general concepts of the degree of freedom and degree of constraint and also gives a geometric explanation of singular cases (see figs. 9-12).

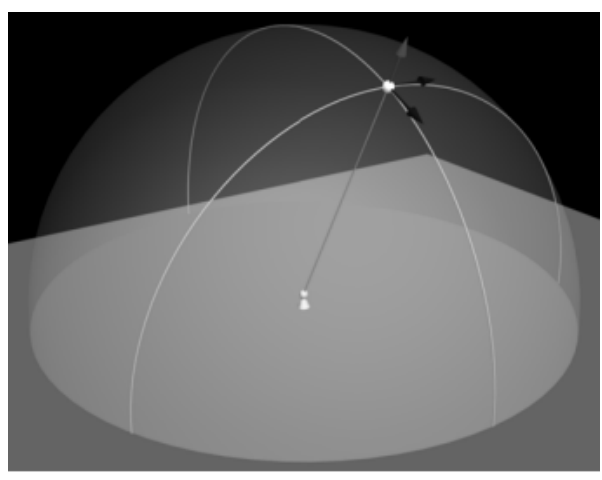

Fig. 9

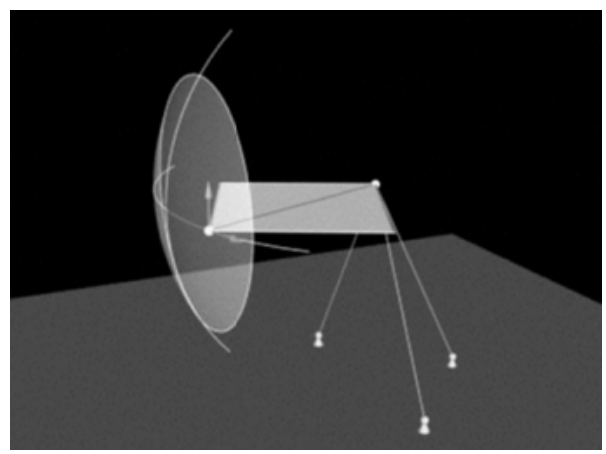

Fig. 11

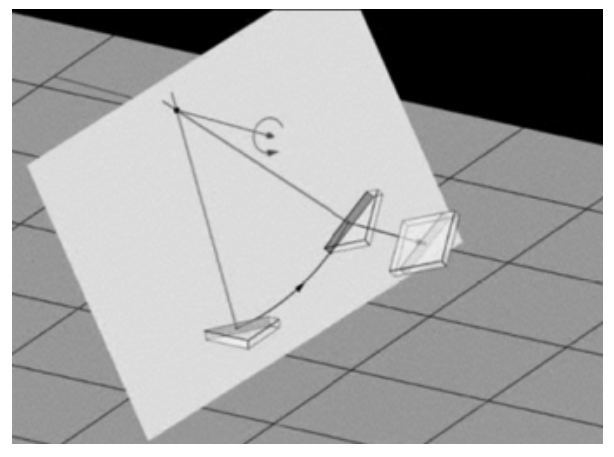

Fig. 10

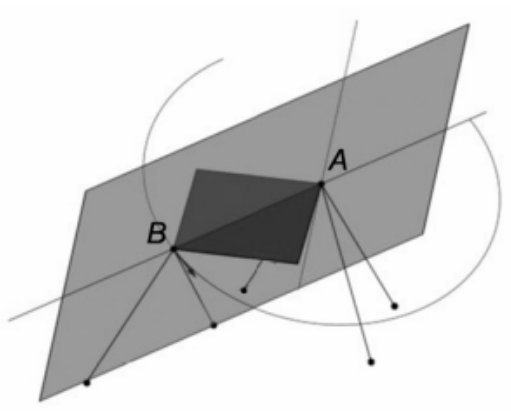

Fig. 12

5.2.2 Algebra. On the other hand, a geometric approach to the problem only will not permit one to confront every planned design situation and, in some cases in particular, one can not do without the assistance of an algebraic formulation.

Therefore, the geometric model prompts the student to propose questions for which there are no easy answers. And at this stage, the student views the film on algebra (still presented using computer graphics) and discovers that in effect, the geometric questions already raised as well as other general questions, can be formulated algebraically.

In particular, here we show an example for which is given the algebraic representation (the equation) of the rigid velocity field, starting from a visualization (simulation in 3D) of the rigid motion and transplacement. This passage is in substance a procedure of linearization, typical and fundamental in structural mechanics, which is introduced utilizing instruments of computer graphics rather than that of formal mathematics. The procedure to obtain the expression

$$
\mathbf{v}(p)=\mathbf{v}(q)+\mathbf{w} \times(p-q)
$$




\section{is the following:}

- A rigid transplacement is shown (simulated);

- Its characteristics are highlighted;

- The velocity is introduced (linearization);

- The formula is constructed.

But let's see in more details

The film (Fig. 10) shows that a rigid transplacement can be decomposed into the product of a rotation around an axis and a translation parallel to it (rotation) (see fig. 13). Since the translation permits an immediate representation, the student's attention is focused on the rotation, stressing that the $3 \mathrm{D}$ problem can be substantially reduced down to a $2 \mathrm{D}$ one (see figs. 14-15).

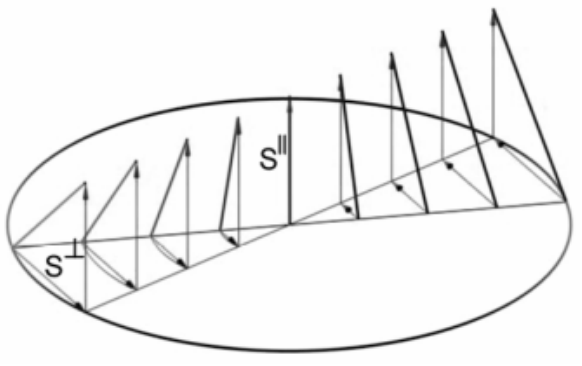

Fig. 13

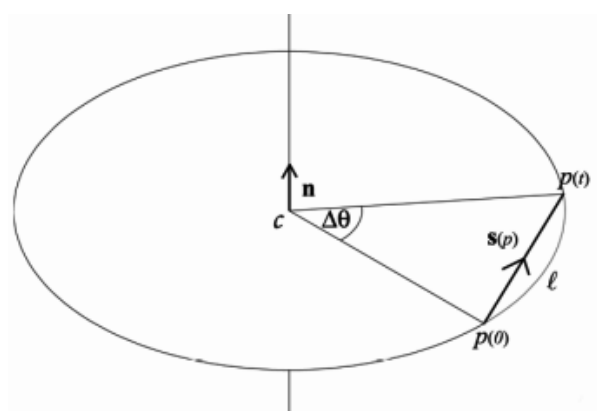

Fig. 15

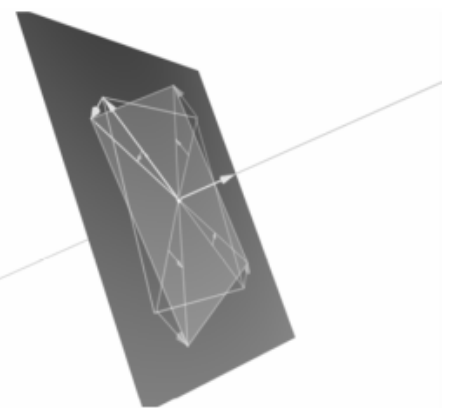

Fig. 14

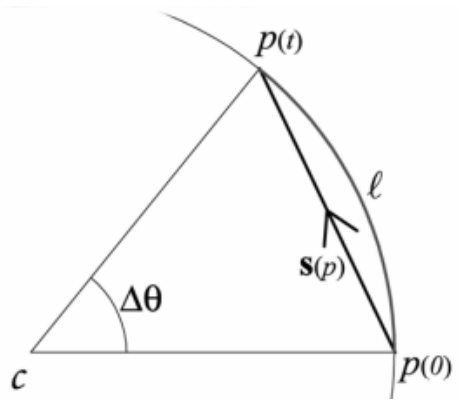

Fig. 16

It should be noted that in the rotation the points of the body move along circumferences and that the length of the arc described in a time $\Delta \mathrm{t}$ by a point $\mathrm{p}$, is directly proportional to the radius $\mathrm{r}$ and to the angle of the rotation $\Delta \theta$. To be precise:

$$
\ell=r \Delta \theta
$$

In order to study motion, let us now pass to the question of velocity. Before introducing vector magnitudes, it is possible to formulate the intuitive concepts of average point velocity, $v^{m}(p)$, and average angular velocity, $w^{m}$, as follows:

106 NicOla L. RizZI AND VALERIO VARANO - Leo: A Multimedia Tale of Structural Mechanics 


$$
v^{m}(p)=\frac{\ell}{\Delta t} \quad w^{m}=\frac{\Delta \theta}{\Delta t}
$$

Therefore, utilizing equation (2), one obtains:

$$
v^{m}(p)=w^{m} r
$$

The preceding description can now be enriched by introducing vector notation. The vectors corresponding to those defined above are:

$$
v^{m}(p)=\frac{\mathbf{s}(p)}{\Delta t} \quad w^{m}=w^{m} \mathbf{n}
$$

Where $\mathbf{s}(p)=p(t)-p(0)$ and $\mathbf{n}$ is a unit vector along the axis of rotation. Naturally, it is important to point out to the student that in general, as $\left\|s^{m}(p)\right\| \leq \ell$ then $\left\|v^{m}(p)\right\| \leq\left|v^{m}(p)\right|$.

A short film shows what happens when $\Delta t \rightarrow 0$. Progressively decreasing $\Delta t$ and at the same time zooming in, it is possible to highlight two fundamental facts: for very small intervals the point displacement is merged with its trajectory; and it also has a perpendicular direction with the radius.

The first fact states that (4), also gives a relationship between instantaneous velocities. Using vectors it can be written in the following form:

$$
\|\mathbf{v}(p)\|=w\|(p-c)\|
$$

where $c$ is the center of rotation.

$$
\text { In effect, for } \Delta t \rightarrow 0 \quad \| \mathbf{s}(p)||=|\ell| \text { so } \| v^{m}(p)||=\left|v^{m}(p)\right| \text {. }
$$

The second fact also gives important information on the direction of the velocity.

The next step is to embed the rotation in the 3D space (see fig. 16), highlighting the fact that the velocity is perpendicular not only to the radius, but also to the vector $\mathbf{w}$ and, therefore, both factors together appear in equation (6). At this point, one takes note of the properties of the cross product and immediately modifies equation (6) to :

$$
\mathbf{v}(p)=\mathbf{w} \times(p-c)
$$

Then one can rewrite equation (7) to obtain the velocity of another point $q$ and subtract the two to obtain equation (1).

It is clear that this presentation tries to extract, as much as possible, the maximum information from the finite motion, which is more easily visualized, to pass to a linear representation that is instead more easily formulated thanks to simple vectorial operations and therefore more useful from an operational point of view.

\subsection{The Design Implications}

5.3.1 Simple design. In the design process, one can face structural problems that can easily be solved by using simple geometric instruments. 
These, despite their simplicity, allow the formulation of a wide range of solutions. Thus knowledge of mechanics can be a guide and stimulation for the design, rather than an obstacle or a limitation.

In the example, one sees that the initial idea of blocking three points $A, B, C$ on a rigid body with three constraints composed of pendulums: a tripod on $A$, a trestle on $B$ and a simple pendulum on $C$, can suggest a number of structures apparently very diverse from one another. To avoid singular cases it is enough to verify that:

- A,B,C are not aligned;

- directions of 3 tripod pendulums are independent;

- trestle doesn't belong to a plane through $A$ and B (see fig. 12);

- single pendulum doesn't belong to a plane trough $A, B, C$.

The structural considerations can interact in a creative manner with considerations both of architectural and functional. For instance one can ask: "how is it possible to aggregate the constraints without excessively limiting the space under the roof?" or "how is it possible to create, by means of the aggregations, an interesting space?" or "what type of symmetries of constraint enrich the architectural project without making it a singular system?”

The range of solutions can be redoubled from a simple observation: the same structural diagram, obviously, works if the three constraints are put 'upside down'(see figs. 17, 18).

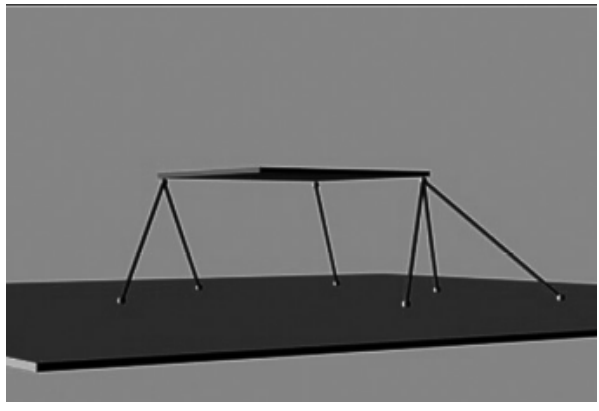

Fig. 17

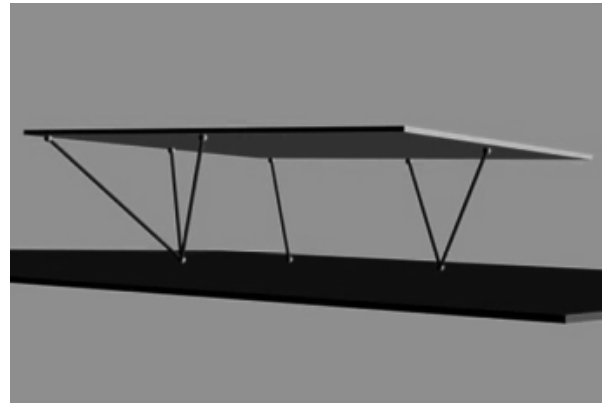

Fig. 18

An alternative structure which is easily found to be isostatic by means of geometric methods is the one made up of three reverse trestles (and the corresponding three $v$ 's) (see fig. 19).

5.3.2 Complex design. In general, anyway, geometric instruments are not sufficient to face more complex design problems, which instead can easily be resolved utilizing algebraic formulation.

In the example, it is noted that the design could be freer than seen thus far: even 6 pendulums with generic directions, applied in 6 different points of the body could block all of the degrees of freedom. In this case; however, a geometric control on the singularity of the system becomes very difficult.

One then introduces the concepts of Lagrangian equations of a constraint, a kinematic matrix and kinematic classification, which, utilizing the algebraic model given above, allow the designer to overcome the impasse.

108 NiCOLA L. RIZZI AND VALERIO VARANO - Leo: A Multimedia Tale of Structural Mechanics 


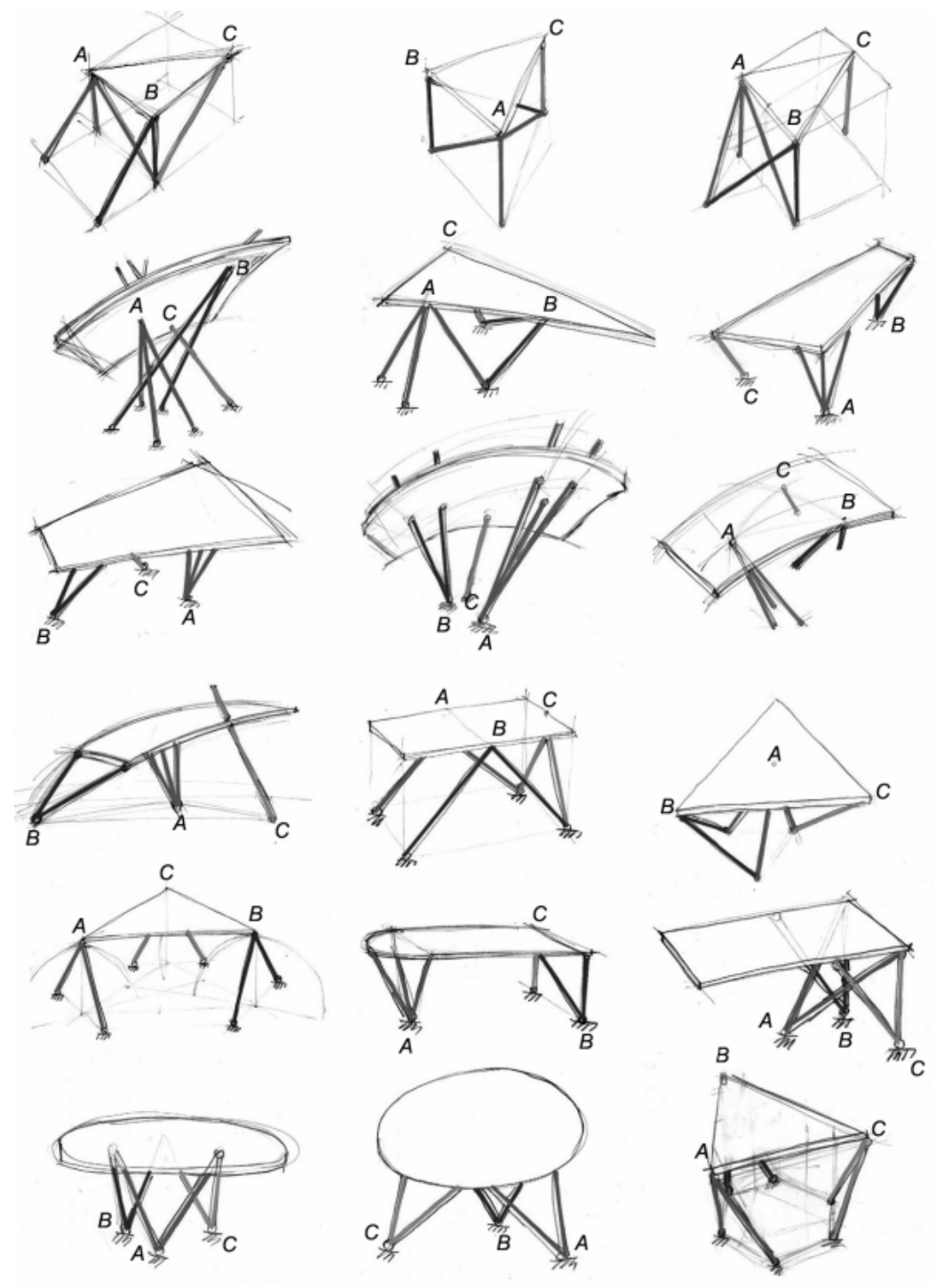

Fig. 19

NEXUS NETWORK JOURNAL - VOL. 7 NO. 1, 2005109 


\section{Conclusions}

In conclusion, we believe that a treatment like this one proposed here can transmit at least two important concepts to the students:

- Taking a problem as a point of departure, the better and deeper the comprehension of the problem, the greater the freedom the designer has to solve it;

- A deeper understanding is possible only by "full immersion" of real objects in "model space", and a thorough study of such models.

\section{Notes}

1. Ludovico Quaroni, Progettare un edificio, Mazzotta 1977.

2. Lucio Russo, La rivoluzione dimenticata, Universale economica Feltrinelli, Saggi, 2001.

\section{About the authors}

Nicola Luigi Rizzi is professor of Structural Mechanics in the Faculty of Architecture and head of the Department of Strucutures in the University 'Roma Tre'.

Valerio Varano graduated in Architecture in 2003 and now is a Ph.D. students in Civil Engineering Sciences, University of 'Roma Tre'. 